\title{
Mujer contra mujer: misoginia femenina en comentarios de noticias en Facebook
}

\author{
Woman vs. Woman: female misogyny in Facebook news \\ comments
}

\author{
Miranda Bustamante, M. A. y Agudelo Vizcaíno, M. F. ${ }^{1}$ \\ Recibido: 13-05-2021 - Aceptado: 17-08-2021 \\ https://doi.org/10.26441/RC20.2-2021-A13
}

\begin{abstract}
RESUMEN: Mediante un análisis de discurso cuantitativo, este estudio se propone establecer la presencia de micro y macro agresiones de mujeres contra mujeres en el proceso de construcción social de la noticia, dentro de los comentarios publicados en las páginas de Facebook de los medios de comunicación más usados en Chile durante 2020, en el contexto posterior a la revolución feminista de 2018 y al estallido social en Chile del 2019. La investigación concluyó que un $26,55 \%$ de los comentarios que hacen mujeres en noticias relacionadas con el género femenino, contienen agresiones dirigidas hacia otras mujeres, lo que confirma la idea de la misógina femenina como consecuencia del sistema patriarcal. La mayor parte corresponde a agresiones del tipo otredad (56\%) y, en segundo lugar, a estigmatizaciones (27\%). En su mayoría (33\%) estas ofensas apuntaron al área personal de las víctimas, lo que confirma un rechazo al ser mujer y al estatus social femenino. Por último, los resultados sugieren que el predominio de las microagresiones, que en conjunto suman un $73 \%$, se debe a que son más sutiles y socialmente aceptadas, mientras que las macroagresiones $(27 \%)$, instalan un estigma más visible sobre las mujeres, que suscita reacciones por parte de otros usuarios y, por lo tanto, un potencial castigo social.
\end{abstract}

Palabras clave: redes sociodigitales; feminismo y comunicación; micro y macro agresiones de género.

\begin{abstract}
Using a quantitative discourse analysis, this research aims to identify female micro and macroaggressions against other women within the news social construction process through the comments published on the top sources of news Facebook pages during 2020, in the context after the "feminist revolution" in 2018 and the social uprising in 2019. The investigation concludes that $26,55 \%$ of all female users' comments on women related news, content aggressions towards other women. That confirms the idea of feminine misogyny, as a consequence of the patriarchal system. Most of these discourses correspond to Otherness (56\%) and, in the second place, to Stigmatization (27\%). The majority of these addressed the personal sphere of the victims (33\%), which confirms a general rejection of women and their social status. At last, results suggest that the microaggressions predominance, that add up $73 \%$ as a whole, is due to the fact that they are subtler and socially accepted than macroaggressions $(27 \%)$, which installs a visible stigma on women and motivates more users' reactions and a potential social punishment.
\end{abstract}

Keywords: social media; feminism and communication; microaggressions and macroaggressions.

\footnotetext{
${ }^{1}$ María de los Ángeles Miranda Bustamante es Doctora en Ciencias Sociales, mención Comunicación Social por la Universidad Nacional de Cuyo, Magíster en Literatura por la UPLA y Periodista por la Pontificia Universidad Católica de Chile. Académica Asociada de la Facultad de Ciencias Sociales, Departamento de Ciencias de la Comunicación, Universidad de Playa Ancha (Chile). Sus líneas de investigación abordan temas de Comunicación digital, Narrativas digitales y Medios sociales digitales. mmiranda@upla.cl, http://orcid.org/0000-0002-4475-1602

María Fernanda Agudelo Vizcaíno es Magister en Pedagogía en Educación Superior, con Mención en Innovaciones Educativas y Magister en Dirección y Gestión de Centros Educativos de la Universidad del Mar. Académica de la Facultad de Ciencias de la Salud, Departamento de Fonoaudiología, Universidad de Playa Ancha (Chile) y Directora del Departamento de Fonoaudiología. Sus líneas de investigación abordan temas de Educación Superior en Carreras de la Salud, Metodologías Innovadoras de Enseñanza y Aprendizaje y Tecnologías de la Información y la comunicación. maria.agudelo@upla.cl, http://orcid.org/0000-0002-0577-0210
} 


\section{Introducción ${ }^{2}$}

A menudo las mujeres pueden odiarse entre sí y someterse entre ellas a comentarios sexistas, con una agresividad comparable a la de los hombres machistas. Esto tiende a ocurrir como una más de las consecuencias del orden patriarcal, que propicia la misoginia femenina, es decir, la tendencia de las mujeres a deslegitimarse e incluso odiarse mutuamente, como una muestra del predominio y valorización sociales de lo masculino (Queijeiro, 2017) (Aguado Vázquez, 2005) (Cixous, 1995).

En Chile, a partir del llamado mayo feminista de 2018, que brotó desde las universidades, comenzaron a expresarse abiertamente las demandas de las mujeres, que incluyeron protestas callejeras, paralizaciones, tomas de establecimientos estudiantiles y denuncias masivas de abusos sexuales, que fueron decantando hacia una propuesta social integradora que reivindicara los derechos de las mujeres, conculcados sistemáticamente por el patriarcado, las instituciones, el capitalismo (Oviedo, 2019). Estas movilizaciones, junto al estallido social del 18 de octubre de 2019, son la cúspide de un nuevo momento generacional liderado por jóvenes que, en los últimos diez años, han demandado igualdad, transformándose en una actoría relevante en el país (Zarzuri, 2020).

Este marco social puede explicar la drástica baja de confianza en los medios tradicionales y analógicos, según los datos del Digital News Report de 2020 (Newman et al., 2020). El informe afirma que Chile es el segundo país del mundo con la mayor caída en la confianza en las noticias emitidas por los medios tradicionales (un 15\% menos que en 2019). Por ello, los medios online y las redes sociales superaron a la televisión como fuente de noticias y Facebook encabeza la lista de las plataformas más usadas en busca de información.

Dentro de este panorama, es posible inferir que la misoginia femenina pueda ser observable en las conversaciones sobre lo público, particularmente en los procesos de reconstrucción social de la noticia que se han masificado gracias a los medios sociales, considerando que lo digital, además de la instantaneidad, permite una potencial incivilidad (Megarry, 2014), la expresión libre de las emociones y también la agresividad en las narrativas de los comentarios de las noticias.

Por todo lo anterior, se plantea como objetivo analizar las narrativas utilizadas por mujeres al comentar noticias vinculadas al género femenino, para identificar la presencia de misoginia femenina en la construcción social de la noticia en Facebook, a través de la caracterización de micro y macro agresiones en los discursos de mujeres contra mujeres.

\section{Marco Referencial}

\subsection{La misoginia femenina}

"Ellos han cometido el peor crimen contra las mujeres. Las han arrastrado, insidiosa, violentamente, a odiar a las mujeres, a ser sus propias enemigas, a movilizar su inmenso poder contra sí mismas, a ser las ejecutoras del viril trabajo". De esta manera Cixous (1995, p. 21), desde el feminismo de la diferencia, describe la llamada misoginia femenina. Sostiene que es consecuencia del patriarcado, en cuanto las mujeres también tienden a aspirar a lo masculino y a valorar a los hombres más que a otras mujeres, que aparecen como desprovistas de valor. Esto ocurre porque, según Lorde (1984), las tácticas del hombre opresor están incrustadas en el interior de las mujeres, por lo que desconfían de sí mismas y suelen aplicar las tácticas masculinas en la forma de relacionarse con sus pares.

\footnotetext{
${ }^{2}$ La presente investigación se realizó en el marco del proyecto CSAL-2020, adjudicado a las autoras en el marco del Concurso Regular de Investigación de la Universidad de Playa Ancha, Valparaíso, Chile.
} 
Esto se cristaliza también en la visión de la sexualidad de Freud (1976), según la cual los niños suponen que todos los seres humanos tienen su mismo aparato genital, por lo que se sorprenden cuando observan el "pene faltante" en el cuerpo femenino. Por su parte, la mujer comienza a envidiar el pene y comienza a desear ser un muchacho. Así también se podría explicar el explícito rechazo a todo lo femenino, comenzando por el cuerpo y continuando con el género.

Este menosprecio de las mujeres por sus pares, se mostró con claridad durante muchos siglos en los salones de las clases altas, donde ellas competían por quien pudiera desposarlas, porque era la única forma de brillar en la sociedad. Así se coló el odio entre mujeres, buscando capturar al mejor de la manada (Queijeiro, 2017).

En la clase trabajadora, en cambio, la industrialización contribuyó a que la mujer, cuando se integró a la producción fabril, asumiera actitudes que históricamente habían formado parte del mundo masculino. Esto la llevó a un forzado cambio psíquico que hasta hoy incluye la "cefalización de los sentimientos" (Aguado, 2005), p.175), es decir, usar la racionalización como método de defensa en los procesos afectivos. De esta manera se forja "un engendro", que habita dentro de la mujer, que aguanta el llanto y el miedo para no parecerse a las demás. "Es un engendro que asume a priori que las rebeldías o el dolor, el cansancio, las necesidades de las mujeres son una performance femenina, teatro victimista-feminista, "pura manipulación" (Aldunate Morales, 2011, s.p.). De esta manera, las mujeres repudian a las que aparecen como víctimas y las culpan de sus desgracias.

En esta situación, las mujeres tienden a hacerse un poco malvadas. De esta manera, la maternidad y el dolor que conlleva, se convierten en criterio de entrada al sexo femenino y de este modo condenan a todas las mujeres a cargar con el sufrimiento como algo propio de la especie. Así lo explica Irigaray (1992), una de las precursoras del feminismo de la diferencia:

Si este sufrimiento se convierte en la norma exclusiva para hacerse mujer, acaba por justificar el sufrimiento en las relaciones amorosas, el sufrimiento moral femenino, etc. Todo ello con el beneplácito de su «masoquismo» y de su capacidad de aguante. A decir verdad, la cultura actual no les deja otras muchas salidas. Masoquista es aquel que vuelve contra sí mismo la agresión destinada a otros. ¿Será necesario que las mujeres agredan a su vez? (p.98).

De este modo, es posible encontrar una explicación a la misoginia femenina en el marco de una cultura falocéntrica, aunque, más que el aparato reproductor, la mujer, obligada a competir, envidia lo más valioso que el hombre tiene: su supremacía social. Ya integrada en el trabajo, aprecia la posición milenaria del hombre como sujeto social y desprecia de paso a otras mujeres, a quienes odia porque -igual que ella misma- están confinadas a una otredad radical. De esta manera, la misoginia femenina puede explicar la existencia de macro y microagresiones de género proferidas discursivamente por mujeres contra mujeres.

\subsection{Las micro y macro agresiones a causa del género}

Estas descalificaciones se presentan de diversas formas, pudiendo ser de manera evidente e intencionada o de forma solapada pero recurrente o incluso cotidiana. El ejercicio del poder de un grupo social sobre otro, puede estar cimentado en modelos que aborden las bases teóricas del prejuicio, pues, como indica el fundamento de la Teoría de la Dominancia Social (Pratto et al., 1994), todas las sociedades suscitan el conflicto entre grupos, lo que las lleva a crear mitos de legitimación que sostienen la desigualdad, resaltando la propia superioridad, como es el caso del prejuicio étnico, del nacionalismo, del sexismo, de la meritocracia y del conservadurismo económico-político (Kattari, 2017). Según Goffman (2019), esto se sostiene desde la categorización social, que define y naturaliza estos grupos y los atributos que se relacionan con cada uno de ellos. Así, el extraño que no cumpla con estas características es signado como defectuoso. 
Este tipo de menosprecio se conoce como estigma, que facilita la discriminación, porque marca la inferioridad de las personas, en tanto que son diferentes. Hace alusión a una marca que permanece en el tiempo, que puede apuntar a edad, clase, color, grupo étnico, creencias religiosas, sexo y sexualidad (Callejas Fonseca \& Piña Mendoza, 2005). Este atributo distintivo es señal de que la persona aludida tiene una falla y carencia de ciertas características (Shelton et al., 2010), que justifican un trato discriminatorio. La estigmatización es un mecanismo de agresión a escala mayor, evidente, intencionado, que se aloja en el campo de las macroagresiones. Es así como, basado en estigmas atribuidos a las mujeres, se ha creado un imaginario colectivo capaz de persuadir a sociedades completas del estatus menor que tiene la mujer dentro de la sociedad, cuando el referente es el hombre. De esta forma, en el marco de la misoginia femenina, tal convicción ha escalado a niveles en los que incluso las mismas mujeres promueven esos estigmas, se ajustan a ellos y discriminan a las que pretenden o logran salir de esos límites. Las macroagresiones son entonces formas de agresión que se caracterizan por ser evidentes, intencionadas, arraigadas a prejuicios y estigmas que se han consolidado a lo largo del tiempo y que, pese a ir perdiendo detractores, siguen arraigados de forma inamovible en ciertos grupos sociales. Es así como estas formas de agresión manifiestas, que antes se circunscribían a ciertos ámbitos de la vida social, con la globalidad y la modernidad, se han transformado en parte de todas las esferas de la vida social y rigen las conductas cotidianas. Sin embargo, en la sociedad moderna la macroagresión no es posible sin una base sociocultural de la microagresión, porque nutre la existencia de dispositivos socioculturales, que van transformando estos comportamientos en aceptables (Mejía, 2014). Por el contrario, las microagresiones son expresiones sutiles y cotidianas, incluso a veces no conscientes, verbales o gestuales, que envían mensajes ofensivos a otro individuo, en este caso a la mujer, por razón de su sexo, y que sugieren inferioridad y devaluación o atribuyen roles culturalmente determinados para la mujer, produciéndole incomodidad y daño psicológico a largo plazo (Yang \& Carrol, 2018). Son formas de ataque más sutiles, que pueden incluso carecer de intención o realizarse de forma inconsciente. Sin embargo, son significativamente más frecuentes que las macroagresiones. Vinculadas, al igual que las macroagresiones, a elementos racistas o sexistas, aparentan ser inofensivas y carentes de importancia, sin embargo, el trasfondo psicológico y social que tienen crea un fuerte impacto en la persona agredida y en toda la sociedad (Ortiz Andrade \& Tejada Grijalva, 2017). Asimismo, las microagresiones pueden ser invisibles para quien las comete, quien no se da cuenta de que humilla y amenaza a quien las recibe. De hecho, los microagresores pueden ser vistos como personas justas y honorables que, sin embargo, albergan a un nivel inconsciente un rechazo contra ciertas "minorías", denostadas socialmente por su raza, orientación sexual o por ser mujeres. Estas manifestaciones "invisibles", como no están bajo resguardo ni conciencia, ocurren espontáneamente sin ningún control ni equilibrio en la interacción personal, social ni laboral.

\subsection{Estado de la cuestión}

El estudio de las microagresiones en el contexto digital es minoritario en comparación con su abordaje en los ámbitos no virtuales. En la esfera online, el análisis de este concepto se ha centrado especialmente en el campo de la psicología y los efectos interpersonales que produce. En esta línea, son frecuentes las indagaciones en las microagresiones raciales. Es el caso de la investigación de Williams et al. (2016), que establece que quienes han sufrido experiencias de discriminación racial en su vida off line tienden a identificar más las microagresiones racistas en los memes que revisan durante sus rutinas virtuales. Posteriormente, el desarrollo de la Perceived Online Racism Scale (PORS) permite evaluar la percepción de los usuarios acerca del racismo en las interacciones interpersonales y la exposición a contenido racista en internet (Keum \& Miller, 2017). Esta forma de medición ha sido legitimada en su utilidad para medir esta percepción sin sesgos (Keum \& Miller, 2018).

En cuanto a los efectos sociales de estas microagresiones, el estudio de Snyder-Yuly (2017) establece 10 categorías de estigmatización racial observadas en las publicaciones de medios de comu- 
nicación estadounidenses y en los comentarios de sus seguidores, que reflejan ideas y estereotipos racistas inconscientes, solapados detrás de un discurso que defiende la equidad.

En general, hay acuerdo en la discusión científica en que las plataformas digitales han permitido más oportunidades de expresión al acoso y los asaltos verbales que constituyen estigmatizaciones. En este sentido, los memes han sido estudiados como dispositivos que perpetúan los estereotipos discriminatorios hacia minorías raciales, a través de su instantaneidad y facilidad de su distribución. Por ejemplo, se ha analizado la caricaturización que hacen de los chinos migrantes en México (Lisbona Guillén \& Rodríguez Balam, 2018). Existen diversas investigaciones, como contraparte, que exploran los medios sociales digitales como herramientas poderosas para promover la inclusión e incrementar la conciencia sobre estas microagresiones, ya sea raciales (Mwangi et al., 2016) (Matías \& Grosland, 2016) o las que afectan a las personas transgénero y otras minorías sexuales (Ingram et al., 2017). Es menor la porción de estudios relacionados con las mujeres y las microagresiones digitales. Uno es el artículo publicado por García, Ruiz \& López-Caniego (2019), que abordó la evolución de la sección micromachismos, abierta por un diario español para recoger denuncias acerca de estas prácticas. Pudieron constatar un incremento sostenido en los aportes de los usuarios, aunque no todos apuntan a micromachismos ni a contenidos mediáticos, sino a todo tipo de abusos patriarcales especialmente en el ámbito privado. En esta misma línea, las microagresiones de los medios de comunicación hacia mujeres atletas en Estados Unidos es el foco del estudio de Kaskan y Ho (2016). Los resultados sugieren una baja cobertura noticiosa de las deportistas en relación con sus pares masculinos y una preeminencia de contenido acerca de su apariencia física más que de sus logros, además de mencionar que algunas no calzan en el molde tradicional de femineidad. Esto, según plantea el estudio, tiene efectos perjudiciales en las atletas a nivel biológico, cognitivo y conductual. Pero también incide en la autoimagen y condición física de las mujeres no atletas que constituyen el público de estos contenidos.

En cuanto al concepto de misoginia femenina o, más ampliamente, del odio entre mujeres, se encontraron solamente algunas investigaciones recientes que tangencialmente rozan en el tema.

Un pequeño puñado de estudios aplicó el concepto al análisis literario, como parte de la interpretación feminista de la diégesis. Es el caso de Rajakannan (2017), quien menciona la misoginia femenina a propósito de la protagonista de Sultana's Dream, escrita por Rokeya Sakhawat Hossain. Este personaje, al adoptar los roles masculinos tradicionales, recibe las críticas de otras mujeres.

También desde la literatura, Faita (2018) defiende la tesis de un feminismo precoz en las Metamorfosis de Ovidio, poema escrito en la Edad Media. En esa línea, descubre rasgos primitivos de misoginia femenina en los personajes, cuando se culpa a las propias mujeres por haber sido víctimas de violación. Interpreta que, en la obra, la corrupción patriarcal fomenta un desprecio de las mujeres hacia sus pares y la división social entre ellas, lo que perpetúa el abuso.

Desde los estudios de género, se hallaron escasas publicaciones, que también abordaron sólo indirectamente la misoginia femenina. Es el caso de la investigación acción con el grupo Tomadoras de Decisiones, que se aplicó en Nuevo León, México, y que estudió, en esa comunidad, el comportamiento de las mujeres agrupadas y su manera de vincularse con sus lideresas. Se concluyó en este aspecto que las mujeres han aprendido de los hombres a ejercer autoridad, porque ellos la han tenido históricamente. Además, se explicó que, mientras a ellos se les ha enseñado abiertamente a competir con sus pares, a ellas se les inculcó que no debían pelear con otras niñas, generando a cambio formas destructivas de rivalidad y de tipo pasivo-agresivas.

Un enfoque similar tiene el trabajo de Sepúlveda Pardo (2020), quien revisó las condiciones impuestas por la pandemia en Chile y su relación con la violencia hacia las mujeres. Con respecto a la misoginia femenina, a través de entrevistas a expertas en políticas públicas y a mujeres 
residentes en la Región Metropolitana, llega a afirmar que el odio y la separación entre ellas se presenta como un obstáculo para el feminismo, que debe resistir esta división, que es producida por el sistema patriarcal. De este modo, se valora la capacidad de organización femenina como signo de resistencia.

Por último, podemos mencionar el estudio que elabora un perfil de las jóvenes fans del heavy metal, que establece que estas mujeres están lejos de querer adoptar sin más las características tradicionales masculinas. Más que eso, buscan la libertad de construir críticamente su propio género, con el poder y autonomía que les inspira ese tipo de música. Por ello, se rehúsan también a verse como las víctimas del sexismo y a caer en la misoginia femenina.

No se encontraron estudios que consideraran el fenómeno de la misoginia femenina relacionado con las microagresiones ni en el ámbito digital.

\section{Metodología}

Estudio descriptivo y transversal de metodología cuantitativa. Se usará el análisis de discurso, que puede ser cuantitativo si se usan datos discretos que se obtienen a través de indicadores que establecen un valor para las características discursivas de los textos de las unidades de análisis, luego de un proceso de interpretación teórica (Sayago, 2014), que proviene, en este caso, de la contextualización del discurso como práctica social (Upton \& Cohen (2009). De acuerdo con el objetivo, se medirán indistintamente la frecuencia de macro y micro agresiones presentes en los discursos de mujeres contra mujeres en los comentarios en Facebook de las noticias que aluden al género femenino.

\subsection{De entre las agresiones, se aplicarán las siguientes variables}

\subsubsection{Foco de la agresión}

Área de desempeño de la víctima donde se concentra la temática la agresión. En esta variable, el primer indicador midió la frecuencia de agresiones por foco, de acuerdo con las siguientes categorías nominales. Las primeras cinco están basadas en los ámbitos de agresiones de la encuesta de la Gender Microaggressions Scale de Capodilupo y Torino, sistematizada por Judson (2014), y orientadas en el presente estudio para su aplicación en textos escritos. Las dos últimas son de elaboración propia y fueron añadidas de forma inductiva luego de revisar el corpus:

3.1.1.1. Apariencia corporal: Se agrede en función de características propias de la corporalidad de la persona, en tanto peso, estatura, fenotipo y vestuario.

3.1.1.2. Capacidad discursiva: La ofensa se basa en el desacuerdo con los argumentos y las opiniones vertidas por otros, independientemente de si su finalidad es persuadir o solo opinar.

3.1.1.3. Rol personal: Se apunta al rol que la víctima cumple dentro de la sociedad en función de su estatus socioeconómico, lugar de habitación, tendencia política, situación familiar, género.

3.1.1.4. Rol laboral: El discurso se dirige al desempeño laboral de la persona, invalidándola en las acciones que competen a su labor profesional $u$ oficio.

3.1.1.5. Capacidad intelectual: La agresión versa sobre la supuesta capacidad intelectual y habilidades mentales del destinatario.

3.1.6. Ética: Se refiere a las conductas y opciones de vida de la persona, a las que se les aplica un juicio valórico. 


\subsubsection{Tipo de agresión}

Se identificaron los tipos de agresión en el marco del ejercicio del poder expresado en el discurso, aun tratándose de mujeres agrediendo a mujeres. Como indicador, se midió la frecuencia de las siguientes categorías, que se detallan en la Tabla 1, agrupadas en macroagresiones, de acuerdo con su connotación siempre descalificadora, y microagresiones, que tienden a ser menos invalidantes y visibles porque están naturalizadas en la cultura.

Tabla 1. Tipo de Agresión

\section{Microagresiones}

Prejuicio: Son actitudes que se refieren al aspecto afectivo ya sea positivo o negativo. La mayoría de las personas utilizan el término prejuicio desde actitudes negativas. Smith y Mackie (1997) indican, al estar enraizados profundamente en las normas sociales de una cultura, facilitan que las personas los aprendan de forma natural, como parte de su crecimiento y conducta (Villegas \& Castañeda, 2017).

Otredad: No supone ninguna diferencia innata o esencial, sino que es una construcción social, resultante de sistemas de etiquetamiento y clasificación (Bayón \& Saraví, 2019). Está configurada y desconfigurada por los discursos del poder y de las políticas públicas que además de establecer diferencias y dualidades generan una opresión, una hegemonía que se traduce en someter y dominar al que es distinto o que no se adecua a los parámetros de valor del sistema capitalista organizado (Montánchez et al., 2017).

Estereotipos de género: Corresponden a construcciones sociales que se aprenden e involucran las definiciones y roles asociados a ser hombre y mujer. Actúan como prescripciones, generando en el individuo la percepción de expectativas sociales de carácter normativo acerca del comportamiento acorde a su género (Oberst et al., 2016).
Macroagresiones

Estigma: Es un atributo profundamente desacreditador en las interacciones sociales, que deshabilita a quien lo recibe para una inclusión plena en la sociedad. Según Goffman (2019), lo fundamental es la connotación social que tiene, ya que puede desacreditar o no a un individuo según la connotación que tenga en el contexto social en que él se encuentre (Vidal Pollarolo, 2018).

Fuente: Elaboración propia.

\subsection{Diseño muestral}

Se analizaron 60 noticias en que se aludía a mujeres. Podían ser protagonistas, víctimas o fuentes, siempre y cuando la alusión se hiciera en el texto de la publicación en Facebook. Las noticias se extrajeron de los 6 medios de comunicación que más utilizaron los chilenos para informarse en 2020, según el Digital News Report (Newman et al., 2020): 24 Horas, Mega, El Mostrador, El Mercurio, Bío Bío y Las Últimas Noticias. Se escogió aleatoriamente la fecha 15 de octubre de 2020 y, desde entonces, se recolectaron de forma retroactiva para cada página de Facebook las últimas 10 noticias que cumplieran las condiciones señaladas. Posteriormente, se utilizó la herramienta Comment Exporter, que permite descargar todos los comentarios de cada publicación con sus respectivos autores y datos asociados.

Esta recolección arrojó un total de 10.997 comentarios. Sin embargo, se detectó que existía una cantidad de textos duplicados, emitidos por el/la mismo autor/a a distintas horas, probablemente por la acción de bots $^{3}$ (Zarel et al., 2020). Estos fueron eliminados, lo que arrojó una muestra definitiva de 10.142 comentarios, de lo que se calculó un promedio real de 169 comentarios por noticia, lo que implica una interacción media considerable.

A continuación, se distinguieron los perfiles de mujeres, versus hombres y otrxs, mediante la identificación convencional del nombre público del perfil o de la verificación del sexo en el perfil en caso

\footnotetext{
${ }^{3}$ Son programas informáticos diseñados para cumplir un propósito específico y producir contenido automáticamente para interactuar con usuarios en las redes sociales, imitando la conducta humana (Zarel et al., 2020).
} 
de ser público. Esto definió un total de 5.292 comentarios de mujeres, que equivalen a un $47 \%$ del total, lo que permite anticipar una división relativamente equitativa de la participación discursiva por sexo en los comentarios de noticias.

\section{Presentación y análisis de resultados:}

Al distribuir las noticias por frentes temáticos, las más comentadas, son las de educación (25\%) y política $(18 \%)$. En el caso de los hombres, los temas más comentados son educación ( $28 \%)$ y política (22\%); en el de las mujeres, son educación $(22 \%)$ y policial $(20 \%)$.

Posteriormente, se analizó discursivamente cada uno de los comentarios proferidos por mujeres para revisar cuáles correspondían a agresiones que específicamente aludieran a una mujer y se hallaron 1.267, que permiten afirmar que más de un cuarto $(26,55 \%)$ de los comentarios de mujeres contienen agresiones contra las mismas mujeres.

Tabla 2. Distribución de Agresiones por Foco

\begin{tabular}{|c|c|}
\hline \multicolumn{1}{|c|}{ Foco } & Porcentaje \\
\hline Apariencia & $2 \%$ \\
\hline Capacidad discursiva & $31 \%$ \\
\hline Ética & $18 \%$ \\
\hline Capacidad intelectual & $12 \%$ \\
\hline Rol laboral & $4 \%$ \\
\hline Rol personal & $33 \%$ \\
\hline
\end{tabular}

Fuente: Elaboración propia.

La mayor parte de estas agresiones, se concentra dentro de la noticia (68\%) y el porcentaje restante se dirige a otras usuarias, que comentan el tema y cuyas intervenciones son criticadas agresivamente en distintos focos, según se aprecia en la Tabla 2, un tercio corresponde al área personal, es decir, al rol específico de la mujer en la sociedad, al estatus que cumple, y constituyen una expresión despectiva hacia la definición canónica del género femenino, a que ciertas mujeres comparten el menosprecio patriarcal hacia las mujeres en la sociedad. En un $31 \%$ se refieren al área discursiva, en que la argumentación es rebatida con violencia por otras interlocutoras, anulando a la emisora más que a sus dichos. Es de interés constatar que las áreas en que tradicionalmente las mujeres han sido agredidas, considerando los estereotipos tradicionales, tienen un bajo porcentaje de agresiones. La apariencia física, la competencia laboral y la intelectual, son las menos frecuentes.

Tabla 3. Distribución de Agresiones por Tipo

\begin{tabular}{|l|r|l|l|}
\hline \multicolumn{2}{|c|}{ Microagresiones } & \multicolumn{2}{c|}{ Macroagresiones } \\
\hline Estereotipo de género & $2 \%$ & Estigma & $27 \%$ \\
\hline Otredad & $58 \%$ & & \\
\hline Prejuicio & $13 \%$ & & \\
\hline
\end{tabular}

\section{Fuente: Elaboración propia}

Como se ve en la Tabla 3, el tipo de agresión más frecuente es la otredad, entendida como una microagresión, que instaura una condición irreal de diferencia, creada culturalmente y que marca una subvaloración y opresión sobre un grupo determinado, en este caso, las mujeres. Inmediatamente después se encuentran las agresiones tipo estigma, al que aludimos como un atributo profundamente desacreditador de acuerdo con la connotación social que se le atribuya y el contexto social en que 
se encuentre (Vidal Pollarolo, 2018). El tipo de agresión evidenciado como menos frecuente es el estereotipo de género, en el que se asigna un atributo a una mujer por el solo hecho de serlo, como resultado de una generalización cultural tradicional.

De esta manera, se puede confirmar la idea expuesta previamente de que las microagresiones, que suman un $73 \%$ en este caso, son más comunes que la macroagresiones $(27 \%)$, porque son más sutiles y pueden pasar inadvertidas, sobre todo si se comparten en un medio social como Facebook.

Tabla 4. Distribución de Agresiones por Sector Noticioso

\begin{tabular}{|c|c|}
\hline Sector Noticioso & Porcentaje de Agresiones \\
\hline Economía & $1 \%$ \\
\hline Educación & $31 \%$ \\
\hline Entretenimiento & $7 \%$ \\
\hline Farándula & $15 \%$ \\
\hline Internacional & $0 \%$ \\
\hline Justicia & $6 \%$ \\
\hline Policial & $24 \%$ \\
\hline Política & $14 \%$ \\
\hline Salud & $1 \%$ \\
\hline Tecnología & $0,3 \%$ \\
\hline
\end{tabular}

Fuente: Elaboración propia.

En cuanto a los sectores o frentes noticiosos, la tabla 4 muestra que la mayoría de las agresiones se concentran en la Educación (31\%), que se remiten a una sola noticia referida a la falta de equidad social en esta materia, que resultó ser la que registró más agresiones y cuya protagonista fue una niña de educación secundaria, etiquetada como de clase alta, que se reunió directamente con el Ministro de Educación para pedir el regreso a clases en tiempos de pandemia. Es importante destacar los resultados de esta unidad de análisis porque, de esas agresiones, un 53\% corresponde a estigma y, por tanto, constituyen macroagresiones. De esta manera, ésta sola noticia contiene el $61 \%$ de las macroagresiones de todo el estudio. El estigma en este caso es revelador en el contexto social de Chile, porque apunta a la edad de la víctima, a su género y a su clase social al mismo tiempo.

En segundo lugar, figuró el área policial (24\%), donde es relevante observar que más de la mitad de las agresiones fueron del tipo Otredad (62\%) y un $28 \%$ de las agresiones en este frente apunta a las mismas mujeres que han sido víctimas de violencia de género, en su gran mayoría (72\%) apuntando al foco ético, culpabilizándolas por no haber sido precavidas o no haber tenido conductas de valor que les permitieran cuidar mejor su vida y su integridad física.

Si miramos el cruce entre foco y tipo de agresión, podemos ver que para todos los focos de agresión la otredad es el tipo de agresión más recurrente, lo que remarca el predominio de esta forma de ofensa. Al revisar cuáles son los focos preponderantes por tipo de agresión, la otredad se da mayoritariamente en el área discursiva (38\%) y personal (27\%), que deshabilitan respectivamente la capacidad argumentativa y el rol social de la mujer.

También es significativo que el estereotipo de género, pese a que fue la agresión menos recurrente, apunte en un 56\% al área ética, lo que permite confirmar que la idea tradicional acerca de la definición y el rol mujer en la sociedad apunta sobre todo a sus decisiones y a sus rasgos valóricos.

En cuanto a la seminalidad, es decir, la capacidad de cualquier publicación en un medio social digital de promover las interacciones de otros usuarios (Miranda Bustamante \& Fernández Medina, 
2020), es posible afirmar que las 1.267 agresiones de mujeres contra mujeres obtuvieron un total de 5.588 reacciones de todo tipo, un promedio de 4,4 por unidad de análisis. Esto implica que las agresiones no fueron indiferentes para los usuarios y usuarias, considerando que las publicaciones del medio de comunicación tienden a ser más visibles por su estatus social y cantidad de seguidores que los comentarios de los particulares (Miranda Bustamante \& Fernández Medina, 2015) y que también, por un asunto de interfaz, para leer y valorar los comentarios de los usuarios, es necesario desplegar los comentarios de la noticia publicada en Facebook y visualizarlos detenidamente, considerando que el promedio de comentarios por noticia fue de 169 .

Además, se estableció que las agresiones emitidas por mujeres y centradas en el aspecto ético de otra mujer son las que generaron más reacciones en promedio $(5,7)$ y las que menos motivaron las reacciones son las agresiones que apuntan a la capacidad intelectual de otras mujeres. En cuanto al tipo de agresión, el estigma obtuvo la mayor cantidad promedio de reacciones por publicación $(6,5)$, y la que menos obtuvo, fue la otredad $(3,4)$. Esto refuerza la idea de que las macroagresiones son más visibles para el público que las microagresiones, que tienden a pasar desapercibidas y al estar naturalizadas.

Dentro del contenido de estas agresiones, si consideramos los sustantivos que más aparecen (hasta 6 veces), llama la atención que un $9 \%$ de los términos más visibles corresponda a distintas formulaciones de "mujer" (mujer, niña, mujeres, señora, hija, madre, etc.). Esto aporta a confirmar que las agresiones se orientan al área personal, a los aspectos de género que implican un rol tradicional y subvalorado de la mujer en la sociedad.

\section{Discusión y Conclusiones}

\subsection{Discusión}

De acuerdo con los resultados, es evidente que la otredad resulta ser la protagonista en el tipo de agresiones, alcanzando, en todos los casos, cifras superiores respecto de los otros tipos. Esto resulta particularmente sensible cuando se emplea un constructo cultural irreal y se instala como elemento desacreditador de otro/a, que distancia al emisor de su interlocutor, a tal punto de desencadenar la agresión. Es llamativo que se direccione la agresión dentro de la noticia, es decir, se ataca a la mujer sujeta de la noticia y con ello también surgen disputas entre las mujeres que comentan, demostrando la escasa tolerancia frente a la diferencia de opinión y especialmente, con la facilidad que otorgan las redes sociales, en este caso Facebook, para poder agredir sin cuidado alguno a otra persona, más aún cuando pertenece a mí mismo género.

La revolución feminista, el estallido social aún sin resolución y las consecuencias sociales y económicas de la pandemia que Chile ha vivido en los últimos tiempos, en su conjunto, han sacado a la luz la frustración y el descontento social del país. Es tal vez por ello que el predominio de los sectores temáticos en que se producen la mayor cantidad de agresiones son el de educación y policial. El descontento y desigualdad en el acceso a la educación refuerza la desigualdad propia que viven las mujeres, y esto contribuye a la polarización de ideas que queda de manifiesto en el análisis realizado. Asimismo, es importante contextualizar que en Chile la inseguridad y los altos niveles de delincuencia, agravados con la crisis económica actual, se han maximizado, redundando el temor especialmente en las mujeres, quienes son víctimas de altas tasas de violencia, alcanzando, por ejemplo, en el año 2020 a 43 casos de femicidios (Sernameg, 2020), en el marco del confinamiento por la pandemia. Nuevamente la inequidad social queda manifiesta en estas discusiones entre mujeres y se suma a la desigualdad de base que sufre el género femenino.

Es importante analizar la diferencia que se pudo establecer entre la agresión por estereotipo y la de 
género, pues su aparición fue escasa y a diferencia de los otros tres tipos, se manifestó mayoritariamente "fuera de la noticia", es decir entre las mujeres que comentaban, más que hacia la mujer que generaba la noticia. Esto puede implicar que como sociedad hemos avanzado en no denostar por atributos asignados culturalmente a las mujeres, al parecer se ha logrado un progreso en cuanto a ello, por lo tanto, su uso ha mermado. Esto pudimos verlo en la escasez de agresiones en el plano de la apariencia física, la inteligencia y la competencia laboral femeninas. Sin embargo, no hay que dejar de lado que esta reducción podría deberse a una forma de no exponerse al juicio social por agredir a mujeres, considerando que la mención explícita de estos estereotipos tiende cada vez más a ser reprochada y castigada en los discursos públicos.

Esto también puede explicar el predominio de la otredad como el tipo de agresión más reiterado, sobre todo centrada en el discurso de otras usuarias mujeres, reforzando la idea de que, aun siendo la emisora también mujer, los planteamientos y argumentos de la aludida distan de los propios, desencadenado la radical separación de opinión, que distancia sustancialmente a un interlocutor, del otro, concluyendo en una serie de agresiones que se agrupan dentro de este tipo de clasificación.

El segundo tipo de agresión más frecuente fue el estigma, a través del cual se instala sobre una persona, en este caso mujer, una condición descalificadora, producto de que no se ajusta a un colectivo social. Estas ofensas tienen como foco principal los atributos personales, es decir, que el hecho mismo de que esa persona sea mujer ya puede invalidarla delante de un determinado grupo social, por no ajustarse a sus convicciones o vivirlas de forma diferente, llevando a la descalificación de ella, por el género al que se adscribe. Aun cuando esta macroagresión se dio un en $27 \%$ de las unidades de análisis, su existencia denota que el ser mujer todavía puede ser una mancha social indeleble y que incluso motiva agresiones que provienen de otras mujeres.

En cuanto a los tipos de agresión por estereotipo de género y prejuicio, coinciden mayoritariamente en enfocarse en la ética de las aludidas, es decir, la disgregación se produce como resultado de un actuar ético aparentemente no compartido, que lleva a presumir que se está transgrediendo la moral, movilizando los límites del comportamiento en un contexto particular. Así, esa diferencia de "normas" conlleva agresiones basadas en creencias sociales preestablecidas y que atienden principalmente a colectivos sociales particulares, que identifican, clasifican y juzgan las conductas de las mujeres de acuerdo con roles y atributos fijados culturalmente en un sistema de género.

En términos generales, tomando en cuenta que la otredad es la agresión mayoritaria (58\%) y el predominio de las microagresiones $(73 \%)$ por sobre las macroagresiones $(27 \%)$, es posible puede inferir que las estigmatizaciones probablemente se inhiban para eludir un castigo social, lo que se comprende al mirar el alto promedio de reacciones obtenidas por cada comentario estigmatizador, en comparación con las otras ofensas, más sutiles, que pueden no ser advertidas por otros usuarios, también porque están naturalizadas culturalmente.

\subsection{Conclusiones}

Este estudio nos permite confirmar la hipótesis de la existencia de agresiones discursivas públicas de mujeres contra mujeres en el proceso de construcción de la noticia, dado que más de un cuarto de los comentarios emitidos por mujeres en relación con otras mujeres constituye una agresión, lo que sugiere la permanencia de la misoginia femenina en Chile, como resabio patriarcal, que se decanta en formas de expresión textuales probablemente facilitadas por el anonimato relativo que ofrece una plataforma como Facebook o por la virtualidad que parece quitarle peso aparente a la agresión verbal en comparación con la que se podría realizar de forma presencial y directa.

Estas agresiones fundamentalmente se dirigen a las mujeres que están insertas en la diégesis de la noticia, ya sea como protagonistas, voceras, testigos, fuentes o víctimas. Esto puede ser un indicio 
de que las mujeres que adquieren cierta notoriedad pública por su trayectoria o por algún acontecimiento contingente, se transforman a menudo en blanco de agresiones verbales de sus pares.

La mayor frecuencia de microagresiones tipo otredad, por sobre la mancha evidente del estigma, también puede interpretarse como un síntoma del estado actual de la mujer. Logrados los derechos sufragistas, laborales y sexuales, la capacidad emancipadora del feminismo funciona hoy "como un motor que va transformando las relaciones entre los hombres y las mujeres y su impacto se deja sentir en todas las áreas del conocimiento" para "percibir las «trampas» de los discursos que adrede confunden lo masculino con lo universal" (Varela, 2008, p.14-15), que deben ser descubiertas con urgencia, porque, desde los discursos, permean la cultura silenciosamente, a tal punto, que ponen a mujer contra mujer.

\section{Bibliografía}

Aguado Vázquez, J. (2005). La envidia del pene. Una reinterpretación a la luz de la Antropología del cuerpo. Anales de Antropología, 39(1), 167-178. http://dx.doi.org/10.22201/iia.24486221e.2005.1.9984

Aldunate Morales, V. (2011, enero 31). Misoginia femenina. Generación 80. http://www.g80.cl/ noticias/columna_completa.php?varid $=11098$

Bayón, M. \& Saraví, G. (2019). Desigualdades: subjetividad, otredad y convivencia social en Latinoamérica. Desacatos. Revista de Ciencias Sociales (59), 8-15. https://bit.ly/2QRzn4q

Callejas Fonseca, L. \& Piña Mendoza, C. (2005). La estigmatización social como factor fundamental de la discriminación juvenil. El cotidiano (134). https://bit.ly/3xmf53E

Cixous, H. (1995). La risa de la medusa: Ensayos sobre la escritura. Anthropos.

Faita, I. (2018). Le féminisme dans les Métamorphoses d'Ovide. Traces d'un féminisme précoce au livre VI. [Tesis de máster, Université Paris Nanterre]. Academia.edu

Flores, L. (2017) (ed.). Mujeres de Nuevo León en el siglo XXI. Editorial Digital Tecnológico de Monterrey.

Freud, S. (1976). Tres ensayos sobre la teoría sexual. Amorrortu.

García, L., Ruiz, M., \& López-Caniego, M. (2019). Micromachismos y prensa digital eldiario. es como estudio de caso. Revista ICONO14 Revista científica de Comunicación y Tecnologías emergentes, 17(1), 162-183. https://bit.ly/32EseY1

Goffman, E. (2019). Estigma: La identidad deteriorada. Amorrortu.

Ingram, M., Speedlin, S, Cannon, Y., Prado, A., \& Avera, J. (2017). A seat at the table: Using social media as a platform to resolve microaggressions against transgender persons. Journal of Creativity in Mental Health, 12(3), 289-304. https://doi.org/10.1080/15401383.2016.1248266

Irigaray, L. (1992). Tú, yo, nosotras. Cátedra.

Judson, S. (2014). Sexist discrimination and gender microaggressions: An exploration of current conceptualizations of women's experiences of sexism [Doctoral dissertation, University of Akron]. ProQuest Dissertations and Theses Global. https://search.proquest.com/openview/7b1bd53c51a0c 3b6b2d0af61 f4bdecbf $/ 1$ ?pq-origsite $=$ gscholar\&cbl=18750\&diss $=y$

Kattari, S. (2017). Development of the ableist microaggression scale and assessing the relationship of ableist microaggressions with the mental health of disabled adults. [Doctoral 
dissertation, University of Denver]. ProQuest Dissertations and Theses Global. https://search. proquest.com/openview/5a6bc3ebb11af6b3118bf585bc033ec9/1?pq-origsite $=$ gscholar \&cbl=1875 $0 \&$ diss $=\mathrm{y}$

Kaskan, E., \& Ho, I. (2016). Microaggressions and female athletes. Sex Roles, 74(7-8), 275-287. https://doi.org/10.1007/s11199-014-0425-1

Keum, B., \& Miller, M. (2017). Racism in digital era: Development and initial validation of the Perceived Online Racism Scale (PORS v1. 0). Journal of Counseling Psychology, 64(3), 310-324. https://doi.org/10.1037/cou0000205

Keum, B. \& Miller, M. (2018). Measurement invariance of the perceived online racism scale across age and gender. Cyberpsychology: Journal of Psychosocial Research on Cyberspace, 12(3). https://doi.org/10.5817/CP2018-3-3

Lisbona Guillén, M. \& Rodríguez Balam, E. (2018). Estereotipos sobre los chinos en México: de la imagen caricaturesca al meme en internet. Revista pueblos y fronteras digital, 13, 1-29. https:// doi.org/10.22201/cimsur.18704115e.2018.v13.3581

Lorde, A. (1984). Sister Outsider. Crossing Press.

Matias, C., \& Grosland, T. (2016). Digital storytelling as racial justice: Digital hopes for deconstructing whiteness in teacher education. Journal of Teacher Education, 67(2), 152-164. https://doi.org/10.1177/0022487115624493

Megarry, J. (2014). Online incivility or sexual harassment? Conceptualising women's experiences in the digital age. Women's Studies International Forum, 47, 46-55. https://doi.org/10.1016/j. wsif.2014.07.012

Miranda Bustamante, M. \& Fernández Medina, F. (2020). Adaptándose a la convergencia: evolución del second screening en Twitter y en programas políticos de TV. Cuadernos. info, (46), 24-46. http://dx.doi.org/10.7764/cdi.46.1708

Miranda Bustamante, M. \& Fernández Medina, F. (2015). Hablándole a la televisión: análisis de las conexiones discursivas entre Twitter y tres programas de contenido político en televisión abierta. Comunicación y sociedad, (24), 71-94. https://bit.ly/3ngRaOs

Montánchez, M., Carrillo-Sierra, S., \& Barrera, E. (2017). Inclusión educativa: Diversidad a partir de la otredad. En Graterol Rivas, M., Mendoza Bernal, M.; Contreras Velásquez, J., Graterol Silva, R. \& Espinosa Castro, J. (Eds.), La Base de la Pirámide y la innovación frugal en América Latina, (pp. 271-282). Ediciones Universidad de Zulia, https://bit.ly/3emof7M

Mwangi, G., Chrystal, A., Bettencourt, G., \& Malaney, V. (2016). Collegians creating (counter) space online: A critical discourse analysis of the I, Too, Am social media movement. Journal of Diversity in Higher Education, 11(2), 146. https://doi.org/10.1037/dhe0000054

Newman, N., Fletcher, R., Schulz, A., And1, S., \& Nielsen, R. (2020). Digital News Report. Reuters Institute. https://reutersinstitute.politics.ox.ac.uk/sites/default/files/2020-06/DNR_2020_FINAL.pdf

Mejía, J. (2014). Corrupción, violencia y cinismo. Notas sobre la insensibilidad moral en el Perú. En Ledezma Narvaez, M. (ed.). Justicia, Derecho y Sociedad. Debates interdisciplinarios para el análisis de la justicia en el Perú (págs. 131-146). Tribunal Constitucional del Perú.

Oberst, U., Chamarro, A., \& Renau, V. (2016). Estereotipos de género 2.0: Auto-representaciones de adolescentes en Facebook. Comunicar, 24(48), 81-90. https://doi.org/10.3916/C48-2016-08 
Ortiz Andrade, A. \& Tejada Grijalva, N. (2017). Campaña de Mercadeo Social” Transforma la Norma microagresiones-macroimpactos". [Disertación de Licenciatura, Universitidad San Francisco de Quito]. Repositorio digital USFQ. https://repositorio.usfq.edu.ec/handle/23000/6591

Oviedo, P. (2019). Ethos colectivo en la movilización estudiantil feminista en Chile o "Mayo Feminista" (2018): del fastidio a la lucha contra la violencia estructural patriarcal. Entornos, 32(2), 20-31. https://bit.ly/3sGeTch

Pratto, F., Sidanius, J., Stallworth, L., \& Malle, B. (1994). Social dominance orientation: A personality variable predicting social and political attitudes. Journal of personality and social psychology, 67(4), 741. https://doi.org/10.1037/0022-3514.67.4.741

Queijeiro, E. (2017). Las hijas de Eva y Lilith: conoce y sana a todas las mujeres que hay en ti. Grijalbo.

Rajakannan, R. (2017). Sultana's Dream: A Cartesian Nightmare of Her Solipsism. Literary Herald, 2(4), 538-547.

Sayago, S. (2014). El análisis del discurso como técnica de investigación cualitativa y cuantitativa en las ciencias sociales. Cinta de moebio, (49), 1-10. http://dx.doi.org/10.4067/S0717$554 X 2014000100001$

Sepúlveda Pardo, A. (2020). Los espacios público-privados y las violencias contra las mujeres: significaciones en contexto de crisis sociosanitaria por COVID-19 [Tesis de licenciatura, Universidad Alberto Hurtado]. Repositorio Universidad Alberto Hurtado.

Sernameg (2020). Femicidios 2020. Sernameg https://www.sernameg.gob.cl/wp-content/ uploads/2021/01/FEMICIDIOS-al-31-de-diciembre-de-2020.pdf

Shelton, J., Alegre, J. \& Son, D. (2010). Social stigma and disadvantage: Current themes and future prospects. Journal of Social Issues, 66(3), 618-633. https://doi.org/10.1111/j.1540$\underline{4560.2010 .01666 . x}$

Smith, E. y Mackie, D. (1997). Psicología Social. Panamerica.

Snyder-Yuly, J. (2017). Modern-Day Minstrelsy: Online Microaggressions and the Digital Narratives of Homeless Black Males [Doctoral dissertation. The University of Utah]. ProQuest Dissertations and Theses Global. https://search.proquest.com/openview/bb9b64d55d899d9927257 7aacf2c1065/1.pdf?pq-origsite $=$ gscholar $\& \mathrm{cbl}=18750 \&$ diss $=\mathrm{y}$

Turbé, S. (2016). Puissance, force et musique metal Quand les filles s'approprient les codes de la masculinité. Ethnologie Française, 46(1), 93-102.

Upton, T. \& Cohen, M. (2009). An approach to corpus-based discourse analysis: The move analysis as example. Discourse studies, 11(5), 585-605.

Varela, N. (2008). Feminismo para principiantes. Ediciones B.

Vidal Pollarolo, P. (2018). La identidad estigmatizada. Polis. Revista Latinoamericana, (3), 1-9. https://bit.ly/3vfXDfC

Villegas, J. \& Castañeda, C. (2017). Estereotipo, prejuicio y discriminación hacia las mujeres en el contexto laboral latinoamericano= Stereotype, prejudice and discrimination against women in the Latin American labor context. Cuestiones de género: de la igualdad y la diferencia (12), 347364. https://bit.ly/3xlUooA 
Williams, A., Oliver, C., Aumer, K. \& Meyers, C. (2016). Racial microaggressions and perceptions of Internet memes. Computers in Human Behavior, 63, 424-432. https://doi. org/10.1016/j.chb.2016.05.067

Yang, Y. \& Carroll, D. (2018). Gendered Microaggressions in Science, Technology, Engineering, and Mathematics. Leadership and research in Education, 4, 28-45. https://bit.ly/2QSzBbq

Zarei, K., Farahbakhsh, R., Crespi, N. \& Tyson, G. (2020). Impersonation on social media: A Deep Neural Approach to Identify Ingenuine Content. arXiv preprint arXiv:2010.08438. https:// bit.ly/3neGZdm

Zarzuri, R. (2020). El malestar de los jóvenes chilenos en el siglo XXI. De la "Revolución pingüina" al "18-O". En De la Fuente, G. \& Mlynarz, D., El Pueblo en Movimiento. Del Malestar al Estallido (págs. 228-246). Catalonia. 